\title{
Spectroscopic characterization of vacuum ultraviolet free electron laser pulses
}

\author{
S. Düsterer, P. Radcliffe, G. Geloni, U. Jastrow, M. Kuhlmann, E. Plönjes, K. Tiedtke, \\ R. Treusch, and J. Feldhaus \\ Hamburger Synchrotronstrahlungslabor (HASYLAB) at Deutsches Elektronen-Synchrotron (DESY) Notkestrasse 85, \\ D-22603 Hamburg, Germany \\ P. Nicolosi and L. Poletto \\ INFM-LUXOR, Department of Information Engineering, University of Padova, Via Gradenigo 6/A, 35131 Padova, Italy
}

P. Yeates, H. Luna, and J. T. Costello

National Center for Plasma Science and Technology and School of Physical Sciences, Dublin City University, Dublin, Ireland

P. Orr

International Research Centre for Experimental Physics, Queen’s University Belfast, BT7 1NN, UK

\author{
D. Cubaynes and M. Meyer
}

LIXAM/CNRS, Centre Universitaire Paris-Sud, Bâtiment 350, F-91405 Orsay Cedex, France

\begin{abstract}
Received January 12, 2006; revised February 21, 2006; accepted March 15, 2006; posted March 23, 2006 (Doc. ID 67229)
Because of the stochastic nature of self-amplified spontaneous emission (SASE), it is crucial to measure for single pulses the spectral characteristics of ultrashort pulses from the vacuum ultraviolet free electron laser (FLASH) at DESY, Germany. To meet this particular challenge, we have employed both photon and photoelectron spectroscopy. Each FEL pulse is composed of an intense and spectrally complex fundamental, centered at a photon energy of about $38.5 \mathrm{eV}$, with a bandwidth of $0.5 \%$ accompanied by higher harmonics, each carrying an intensity of typically 0.3 to $0.6 \%$ of that of the fundamental. The correlation between the harmonics and the fundamental is in remarkable agreement with a simple statistical model of SASE FEL radiation. (C) 2006 Optical Society of America

OCIS codes: $140.2600,140.7240,300.6210$.
\end{abstract}

Free electron lasers (FELs), operating on the principle of self-amplified spontaneous emission ${ }^{1}$ (SASE), open up completely new vistas in intense lasermatter interaction, as they have the potential to provide ultrashort pulses of coherent laser radiation with photon energies far above the ionization thresholds of matter. As a result, nonlinear optics and spectroscopy can be extended into the vacuum ultraviolet (VUV) region of the electromagnetic spectrum for the first time to our knowledge. In contrast with traditional visible lasers, the predominant interaction of VUV-FEL radiation is expected to be with inner-shell electrons. Hence new physical phenomena, in which electrons in resonant autoionizing ${ }^{2}$ and continuum states $^{3}$ play an important role, are expected to be observed.

Established VUV sources, such as storage-ringbased insertion devices and high harmonics of optical lasers, lack the enormous brilliance of the FLASH facility, but they do enjoy stable conditions of operation. In contrast, the statistical character of the SASE process gives rise to intensity and frequency fluctuations for each individual shot. Hence it is absolutely essential to have the capacity to determine the salient properties of the FEL beam, such as spectral distribution and intensity variations, on a shot-to-shot basis. Similar to SASE FELs in the visible frequency range, ${ }^{4,5}$ SASE VUV-FELs emit intense odd ${ }^{6}$ and even ${ }^{7}$ harmonics up to the percent level. The ratio of the third harmonic to the fundamental intensity grows strongly within the linear regime of operation and is limited to a theoretical maximum of $2 \%$ when the FEL output reaches saturation. ${ }^{6}$ To comprehensively characterize the FEL spectral distribution, we combined a high-resolution grazing incidence spectrometer to record single-shot spectra, with a time-of-flight (TOF) photoelectron spectrometer capable of recording electrons produced by the fundamental, as well as the harmonics, of the FEL radiation within one single pulse.

Kinetic energy analysis of the photoelectrons was obtained by using a magnetic bottle electron analyzer similar to that described in Ref. 8. Electrons produced by the interaction of an effusive gas jet and the FEL beam (focus $\sim 30 \mu \mathrm{m}$ FWHM) in the acceptance volume of the analyzer are directed by a strong permanent magnet $(0.5 \mathrm{~T})$ towards a $65 \mathrm{~cm}$ long TOF tube. A solenoid $(0.5 \mathrm{mT})$ then guides the electrons further. The total collection efficiency of this configuration is close to $100 \%$ for all considered kinetic energies. The total photoelectron current was detected with a conventional two-stage channel plate detector. A typical energy resolution of $5 \%$ of the kinetic energy was achieved, which can be improved by decel- 


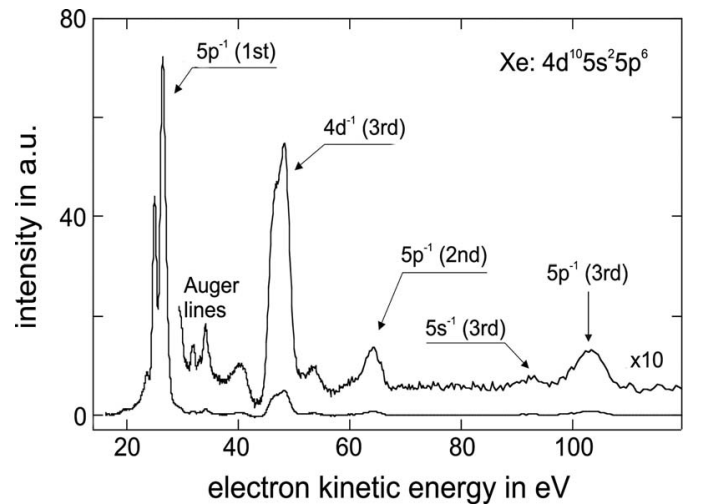

Fig. 1. Photoelectron spectrum of Xe recorded after interaction with VUV photons from the FEL lasing at $32.2 \mathrm{~nm}$ $(38.5 \mathrm{eV})$. The $5 p$ photolines as well as the additional structures induced by the second and third harmonics of the FEL are indicated.

erating the electrons with the aid of an electrostatic retardation field at the entrance of the TOF. Rare gases $(\mathrm{He}, \mathrm{Ar}$, and $\mathrm{Xe}$ ) were introduced through a capillary of $500 \mu \mathrm{m}$ inner diameter. The background pressure was approximately $2 \times 10^{-8}$ mbars without gas and was raised to $2 \times 10^{-7}$ mbars with a rare gas. The linear accelerator was operated with a repetition frequency of $2 \mathrm{~Hz}$ for the macro pulse, consisting of a pulse train of up to 20 individual electron bunches, with $1 \mu$ s separation. The central wavelength of the SASE FEL radiation was about $32.2 \mathrm{~nm}(38.5 \mathrm{eV})$ with a temporal width, estimated from the spectral properties of each pulse to be less than $50 \mathrm{fs} .{ }^{9}$ The average energy of the pulses, monitored by detectors provided by DESY, ${ }^{9}$ varied typically between 5 and $20 \mu \mathrm{J}$ per pulse. These values correspond to $\sim 10^{12}-10^{13}$ photons per pulse.

A typical photoelectron spectrum recorded by summing over 256 individual FEL pulses is displayed for $\mathrm{Xe}$ in Fig. 1. The strongest feature in the spectrum corresponds to the photoionization of the two spinorbit components of the Xe $5 p$ shell $\left({ }^{2} P_{3 / 2}\right.$ and ${ }^{2} P_{1 / 2}$ at kinetic energies of $E_{\text {kin }}=26.4$ and $25.1 \mathrm{eV}$, respectively). Additional structures at kinetic energies $E_{\text {kin }}>30 \mathrm{eV}$ are attributed to the photoionization of the Xe atoms in the $5 p, 5 s$, and $4 d$ shell by the second $[\hbar \omega(2 \mathrm{nd})=77 \mathrm{eV}]$ and the third $[\hbar \omega(3 \mathrm{rd})=115.5 \mathrm{eV}]$ harmonic of the fundamental FEL radiation. In particular, because of the high $4 d$ photoionization cross section the broad peak at $E_{\text {kin }}=47 \mathrm{eV}$, which is assigned to the unresolved $4 d^{-1}$ photolines $\left({ }^{2} D_{5 / 2}\right.$ and ${ }^{2} D_{3 / 2}$ ), and the corresponding Auger transitions around $E_{\text {kin }}=30 \mathrm{eV}$ are clearly visible. It should be stressed that we cannot distinguish between photoionization induced by the higher harmonics of the FEL and direct two- and three-photon ionization, which should become possible for high intensities. We have excluded such nonlinear processes, since almost identical values are deduced from the spectra of all three gases. Since the energy dependence of the partial photoionization cross section for the Xe $5 p, 5 s$, and $4 d$ shell is known with high accuracy, ${ }^{10}$ the integrated intensity of the corresponding lines in the photoelectron spectrum can directly be converted to intensities of the harmonics relative to the fundamental. Average values for both the second and third harmonics were found to be equivalent within the error bars. Ratios of $(0.3 \pm 0.2) \%$ and $(0.6 \pm 0.2) \%$ have been determined for $\sim 5$ and $\sim 20 \mu \mathrm{J}$ FEL pulse energies, respectively.

Because of statistical fluctuations in the SASE process, there may be considerably more harmonic content in an individual FEL pulse than the mean values given above. This question can be addressed by measuring the photoelectron spectra for each single FEL pulse. Due to the large photon flux per pulse and the high rare-gas photoionization cross sections, single-shot spectra were easily recorded. In Fig. 2 the absolute intensity of the $\mathrm{Xe} 4 d^{-1}\left(E_{\text {kin }}=47 \mathrm{eV}\right)$ line versus the $5 p^{-1}\left(E_{\text {kin }}=25-26 \mathrm{eV}\right)$ lines is shown, i.e., indicating the relative intensity of the third harmonic of the FEL with respect to the intensity of the fundamental. The plot shows a strong correlation between a high FEL intensity and a strong thirdharmonic signal. For intense pulses the spread of the data points is quite large. This can be explained by the strong dependence of the third harmonic on intensity spiking in the fundamental, since the intensity of the third harmonic is proportional to the cube of the intensity of the fundamental, $I_{3}(t) \propto\left[I_{1}(t)\right]^{3}$.

A simple theoretical approach was used to simulate correlations between fundamental and harmonic pulse energies (see Fig. 2). In the underlying model we assumed that the SASE FEL radiation can be described in the linear mode of operation, i.e. the exponential gain regime, using Gaussian statistics. ${ }^{11}$ This analytical approach is not valid in the nonlinear regime, at saturation. In our case, where the FEL operates between both regimes, the qualitative, linear approach still represents the fundamental properties well. In the linear region, the instantaneous intensity of the fundamental, $I_{1}(t)$, shows a negative exponential probability distribution $p\left[I_{1}(t)\right]=1 /\left\langle I_{1}(t)\right\rangle$ $\times \exp \left[-I_{1}(t) /\left\langle I_{1}(t)\right\rangle\right]$, where \langle\rangle denotes the average over an ensemble of pulses. The summation over $M$ subpulses yields a reference for one SASE pulse at the fundamental wavelength. $M$ can simply be measured by analyzing the energy fluctuation data ac-

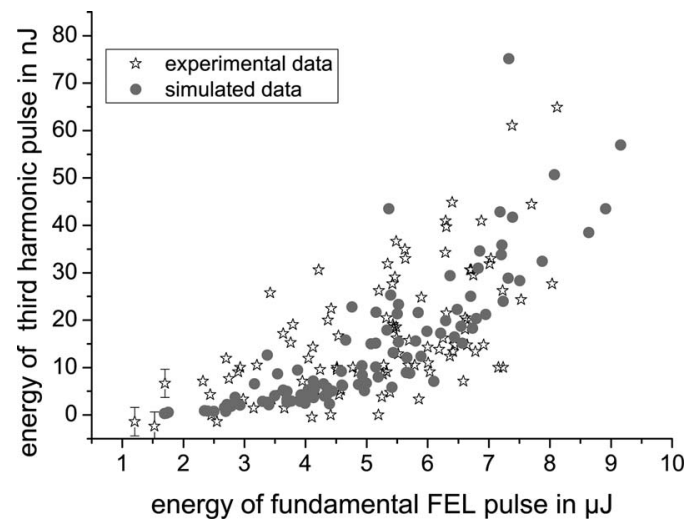

Fig. 2. Relative pulse energies of the fundamental and the third harmonic were measured for 100 individual FEL pulses (stars) by TOF spectroscopy. The simulated SASE pulses (six modes, circles) and the TOF data were scaled to the provided average pulse energy of $\sim 5 \mu \mathrm{J}$. 
quired from the fundamental radiation. ${ }^{11}$ However, summing the cube of the instantaneous intensity for each subpulse represents a relative value for the third-harmonic pulse. To compare the simulated data with the experiment, the average ratio of the simulated third harmonic to the fundamental was scaled to the experimentally determined average. With such a model, we can analyze the individual temporal pulse shapes for all simulated pulses in Fig. 2. Indeed, a high ratio of the third harmonic to the fundamental for one single FEL pulse corresponds to a distinct spike in the time domain of the fundamental and, even more pronounced, in the third-harmonic pulse. ${ }^{12}$ Since the model yields good qualitative agreement with the experiment, we infer that the underlying temporal structure predicted by it must also be a good qualitative representation of the real pulse shapes, which we cannot measure directly.

To investigate the spectral distribution in more detail, high-resolution single-shot spectra (Fig. 3) were recorded in the range $10-40 \mathrm{~nm}$ by a grazing incidence stigmatic spectrometer ${ }^{13}(\Delta \lambda$ $\sim 0.04 \mathrm{~nm}$ at $30 \mathrm{~nm}$ ). The spectrum is acquired on a fluorescent screen (spectral window of $\sim 2 \mathrm{~nm}$ ), that is imaged by a gated intensified camera capable of recording spectra from single FEL pulses out of a bunch train. Spectra of the second and the third harmonic, averaged over 2500 shots, have been recorded (see the inset in Fig. 3). Considering all spectrometer components, the wavelength-dependent response was calculated. The relative intensities of the harmonics to the fundamental are determined as $2 \mathrm{nd} / 1 \mathrm{st}=\left(0.35_{-0.20}^{+0.35}\right) \%$ and $3 \mathrm{rd} / 1 \mathrm{st}=\left(0.4_{-0.25}^{+0.40}\right) \%$ by the spectrometer for an average pulse energy of $\sim 20 \mu \mathrm{J} .{ }^{14}$ Despite the different locations of the detectors, ${ }^{15}$ these values are in good agreement with the photoelectron measurement.

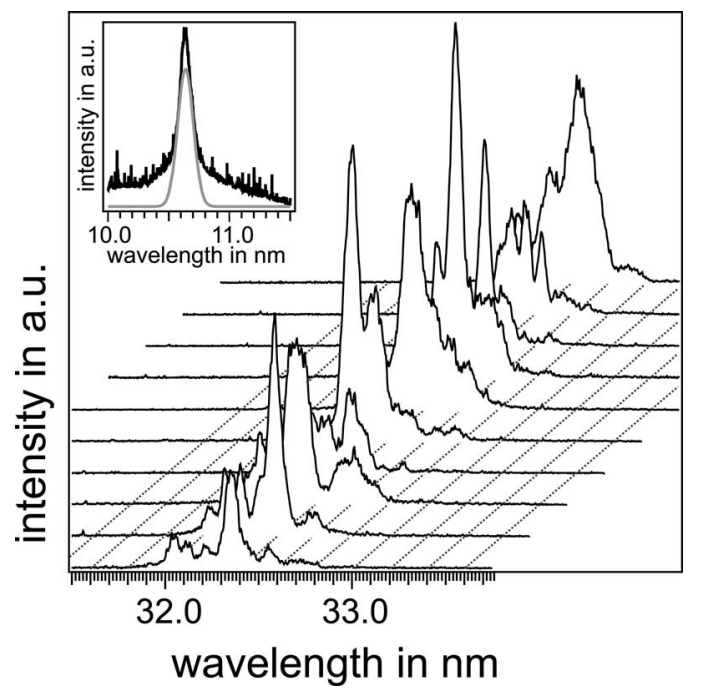

Fig. 3. VUV spectra for ten arbitrarily chosen FEL pulses. The main wavelength for all pulses is about $32.2 \mathrm{~nm}$, with an overall width of about $0.4 \mathrm{~nm}$. Inset, third-harmonic spectrum, centered at $10.7 \mathrm{~nm}$, averaged over 2500 pulses.
In conclusion, we have characterized the spectral properties of the FLASH lasing at a wavelength of $32.2 \mathrm{~nm}$. Using TOF photoelectron spectroscopy, the ratio of the pulse energy of the fundamental and the two higher harmonics within one FEL pulse have been measured on a shot-to-shot basis ( $1 \mu \mathrm{s}$ pulse spacing). Our detection method is nonperturbative, and thus it can be used as an online detector for experiments to deliver information about the harmonic content of the FEL pulses.

We thank the FEL-operator crew for delivering the SASE beam as well as E. Saldin, E. A. Schneidmiller, M. Yurkov, and Th. Tschentscher for helpful discussions. J. T. Costello and P. Orr acknowledge support from the HEA North South Programme, and H. Luna from the EU Marie Curie Programme. Support from the EU RTD-project "X-Ray FEL Pump-Probe" HRPICT-1999-50009 is gratefully acknowledged. P. Radcliffe's e-mail address is Paul.Radcliffe@desy.de.

\section{References}

1. J. Andruszkow et al., Phys. Rev. Lett. 85, 3825 (2000).

2. S. I. Themelis, P. Lambropoulos, and M. Meyer, J. Phys. B 37, 4281 (2004).

3. M. A. Kornberg and P. Lambropoulos, J. Phys. B 32, L603 (1999).

4. V. Sajaev, Z. Huang, S. Biedron, P. Den Hartog, E. Gluskin, K. Kim, J. Lewellen, Y. Li, O. Makarov, S. Milton, and E. Moog, Nucl. Instrum. Methods Phys. Res. A 506, 304 (2003).

5. A. Tremaine, X. Wang, M. Babzien, I. Ben-Zvi, M. Cornacchia, H.-D. Nuhn, R. Malone, A. Murokh, C. Pellegrini, S. Reiche, J. Rosenzweig, and V. Yakimenko, Phys. Rev. Lett. 88, 204801 (2002).

6. E. Saldin, E. Schneidmiller, and M. Yurkov, "Properties of the odd harmonics of the radiation from SASE FEL with planar undulator," Opt. Commun. (to be published).

7. G. Geloni, E. Saldin, E. Schneidmiller, and M. Yurkov, "Exact solution for the second harmonic generation in XFELS," Opt. Commun. (to be published).

8. J. Eland, O. Vieuxmaire, T. Kinugawa, P. Lablanquie, R. Hall, and F. Penent, Phys. Rev. Lett. 90, 530031 (2003).

9. V. Ayvazyan et al., Eur. Phys. J. D 37, 297 (2006).

10. U. Becker and D. A. Shirley, eds., VUV and Soft X-Ray Photoionization (Plenum, 1996).

11. E. Saldin, E. Schneidmiller, and M. Yurkov, The Physics of Free Electron Lasers (Springer, 1999).

12. W. Brefeld, B. Faatz, J. Feldhaus, M. Korfer, J. Krzywinski, T. Möller, J. Pflueger, E. Saldin, E. Schneidmiller, S. Schreiber, and M. Jurkov, Nucl. Instrum. Methods Phys. Res. A 507, 431 (2003).

13. L. Poletto, L. Epulandi, P. Nicolosi, M. Pelizzo, P. Zambolin, J. Feldhaus, U. Jastrow, U. Hahn, E. Plönjes, and K. Tiedtke, in Proc. SPIE 5534, 37 (2004).

14. The main causes of error are (i) the uncalibrated fluorescence yield of the Ce:YAG screen and (ii) the different angular distributions of the fundamental and the harmonics.

15. Since the transmission of the beamline is almost constant over the $30-130 \mathrm{eV}$ photon energy range, only the total FEL intensity is reduced between the spectrometer and the end station, located about 25 and $70 \mathrm{~m}$ behind the undulator, respectively. 